\title{
Patterns of Nestedness in Remote Polynesian Ant Faunas (Hymenoptera: Formicidae) $^{1}$
}

\author{
Lloyd W. Morrison ${ }^{2}$
}

\begin{abstract}
The entire ant faunas of remote Polynesian islands consist of introduced species. An important question concerning the assembly of Pacific island ant faunas is whether these species are a random assortment of the available species pool, or whether they exhibit highly ordered occurrence patterns (i.e., nested subsets of species). I evaluated nestedness for the ant faunas of two island groups in remote Polynesia: (1) the Hawaiian Islands, and (2) French Polynesia and the Cook Islands. Wilcoxon two-sample tests were used to analyze nestedness patterns for individual species and islands; the degree of nestedness for species assemblages and archipelagos was determined by combining tail probabilities of individual species and islands. Both island groups revealed highly significant nestedness at the level of the assemblage (a per-species approach) as well as the archipelago (a per-island approach). Considered individually, most species (73-95\%) and most islands (89-100\%) demonstrated significant nestedness. Instances of nonsignificant nestedness were frequently associated with low statistical power. These results reveal a strong deterministic element in the assemblage of remote Polynesian ant faunas. Dispersal opportunities along with presence of appropriate habitat type are likely the most important mechanisms underlying the observed patterns.
\end{abstract}

The entire ant Faunas of the more remote Polynesian islands (east of Rotuma, Samoa, and Tonga) consist of introduced species. Although most introductions have occurred within the last $200 \mathrm{yr}$, some introductions may date to the ancient Polynesians (Wilson and Taylor 1967a). These islands represent rare opportunities to study patterns of community organization among species that share no common coevolutionary history in the region.

A collection of early survey records suggested that the ant faunas of these islands were depauperate, and that strong interspecific aggression resulted in complementary

\footnotetext{
${ }^{1}$ Manuscript accepted 20 February 2007.

2 Department of Biology, Missouri State University, 901 South National Avenue, Springfield, Missouri 65897 (phone: 417-836-3119; fax: 417-836-8886; e-mail: LloydMorrison@MissouriState.edu).
}

Pacific Science (2008), vol. 62, no. 1:117-127

(C) 2008 by University of Hawai'i Press

All rights reserved distribution patterns (Wilson and Taylor 1967b); this remained the conventional wisdom for many years (Hölldobler and Wilson 1990). A more comprehensive reevaluation based on more thorough collections revealed more species were present than previously thought and found no evidence of mutually exclusive distribution patterns among islands (Morrison 1996a). Ant communities examined on the island of Moorea revealed patterns of structure and function similar to those found in more speciose, coevolved mainland communities (Morrison 1996b).

Here I examine whether the ant species occurring on remote Polynesian islands are a random assortment of the available species pool, or whether they exhibit highly ordered occurrence patterns (i.e., nestedness or nested subsets of species). Nestedness occurs when the species composition of each island represents a subset of the species assemblage on more speciose islands. A high degree of nestedness implies that the geographic distribution of species is a deterministic (and predictable) function of physical, biological, or anthropogenic processes, and this has 
important implications for biogeography, evolutionary ecology, and conservation (e.g., Patterson and Atmar 1986, Patterson 1987, Simberloff and Martin 1991, Cook 1995, Cook and Quinn 1995, Kadmon 1995, Hecnar and M'Closkey 1997, Wright et al. 1998, Hecnar et al. 2002).

The following questions are posed: (1) Are the introduced ant faunas of remote Polynesian islands significantly nested at the level of species assemblages and archipelagos? (2) Which individual species or islands contribute to this nestedness, and which do not? and (3) What are the potential mechanisms of the observed nestedness?

\section{MATERIALS AND METHODS}

\section{Ant Species Records}

Nestedness was evaluated for the ant faunas of two of the more thoroughly collected island groups in remote Polynesia: (1) the Hawaiian Islands and (2) French Polynesia and the Cook Islands. All of the ant species present in both of these island groups are introduced. The main Hawaiian islands of O'ahu, Hawai'i, Kaua'i, Maui, Lāna'i, and Moloka'i are arguably the best-collected islands for ants in Polynesia. Collections of ants have also been made from a number of the smaller Northwestern Hawaiian Islands. For my analysis, I used the list of established species in Krushelnycky et al. (2005) (except for some Cardiocondyla species, see later in this section). Geographical distribution records were obtained from Nishida (2002), with the following exceptions for recently established species: Pheidole moerens and Solenopsis sp. (Gruner 2003), Tetramorium insolens (Kumashiro et al. 2001), and Wasmannia auropunctata (Krushelnycky et al. 2005).

Records of ants from French Polynesia and the Cook Islands were compiled from several sources. Perrault (1988) recorded the ant fauna of Tahiti and that of the atoll Fangataufa (Perrault 1993). The Society Islands of Moorea, Bora Bora, and Huahine were surveyed by Morrison (1996a), as well as five small islands on the barrier reefs of Moorea (Tiahura, Fareone, Ahi, and Irioa) and Bora
Bora (one unnamed island) (Morrison 1995). Morrison (1997) surveyed 10 islands in the Marquesas, Tuamotus, Gambiers, and Cook Islands. Collections from the main Society group of Tahiti, Moorea, Huahine, and Bora Bora were relatively thorough (Morrison $1996 a$ ), as were records from the five small islands on the barrier reefs of those main islands (Morrison 1995). Collections from the other islands in this group were not as thorough (Morrison 1997).

In the records from French Polynesia and the Cook Islands, a few forms were not identified to species. Considering these records as distinct species could artificially increase the species richness in the island group and decrease the degree of nestedness. Thus these forms (five ponerines and one Monomorium sp.) were not included in these analyses. A Brachymyrmex sp. from Tahiti was retained, because no other Brachymyrmex were recorded from French Polynesia or the Cook Islands.

Seifert (2003) revised the Cardiocondyla species groups, changing the identities of Cardiocondyla species known from Polynesia. Seifert did not examine all Cardiocondyla material from these island groups, however. Thus it is not possible in all cases to determine with certainty which species now known and described are present on which islands. Furthermore, distributional records for $\mathrm{Ha}-$ wai'i (Nishida 2002) and French Polynesia are all based on species concepts before Seifert's revisions. For the analyses presented here, I used the species concepts in place before Seifert's revision.

\section{Quantification of Nestedness}

Wilcoxon two-sample tests (also known as Mann-Whitney $U$ tests) were used to analyze nestedness patterns. This approach was first used by Schoener and Schoener (1983) to measure the "occurrence sequences" of species on islands, by ranking islands according to the magnitude of a particular variable (e.g., area) and then determining whether the associated presence/absence sequence of the species of interest was significantly nonrandom. This procedure has since been used to ana- 
lyze nestedness patterns for individual species (Patterson 1984, Simberloff and Levin 1985, Simberloff and Martin 1991, Kadmon 1995, Nores 1995, Hecnar and M'Closkey 1997, Hecnar et al. 2002). Although nestedness is, strictly speaking, a property of a species assemblage rather than an individual species, referring to individual species that have nonrandom occurrence sequences as being "significantly nested" is usually done as a matter of convenience (e.g., Hecnar et al. 2002).

A nested matrix is constructed with species rank-ordered in terms of decreasing numbers of occupied islands, and islands rank-ordered in terms of decreasing numbers of species. If perfectly nested, all cells in the upper lefthand corner of the matrix would consist of "presences" and all cells in the lower righthand corner would consist of "absences." The greater the departure from this pattern in the ordered matrix, the greater the departure from nestedness.

For each species, the Wilcoxon test (onetailed) determines the degree to which the sequence of presences and absences is ordered, compared with a null hypothesis in which presences and absences occur at random. Species that are present on all islands do not provide evidence for or against nestedness and cannot be analyzed with this two-sample test. Statistical power to detect nestedness is low for species that are present on almost all, or very few islands. For small sample sizes, it may not be possible to obtain a "significant" result $(\alpha=.05)$. This is taken into consideration when interpreting the results.

The degree of nestedness for entire species assemblages was determined by assuming that the species are independent and combining the tail probabilities of the individual species. The resulting statistic in this metaanalytic approach is chi-square distributed, with $2 k$ degrees of freedom $(k=$ the number of individual test probabilities) (Sokal and Rohlf 1995). All species (except those that occurred on all islands) were included in these meta-analyses, even those for which individual significant results could not be obtained due to lack of power.

Although previous applications have focused on nestedness as a function of species (individuals and assemblages), this approach could also be used to evaluate nestedness as a function of islands (both individual islands and archipelagos). Once ranked as described here, the Wilcoxon test is applied to the sequence of presences and absences of species on each island; combining tail probabilities of individual islands yields the degree of nestedness for the entire archipelago. Here I use this technique to evaluate nestedness for individual species and entire species assemblages, and also for individual islands and entire island archipelagoes. Analyses were conducted for: (1) 14 Hawaiian islands, (2) 20 islands in French Polynesia and the Cook Islands, and (3) a subset of the nine most thoroughly collected islands in French Polynesia, all of which are in the Society group. SPSS 11.0.4 for Mac OSX (SPSS 2005) was used for all analyses.

\section{RESULTS}

Both archipelagoes revealed highly significant nestedness at the level of the assemblage (a per-species approach) as well as the archipelago (a per-island approach) (all $P<.001$ ). This was true for the expanded French Polynesia/Cook Island group, as well as the more thoroughly collected Society Island subset.

Considered individually, a large proportion of species and islands demonstrated significant nestedness. In the Hawaiian Archipelago, all individual islands were significantly nested (Table 1). On an individual species basis, seven species occurred on only one island and it was not possible to obtain a significant result for these. Of the remaining 38 species, $36(95 \%)$ were significantly nested (Table 1).

In the larger French Polynesia/Cook Islands group, on an individual island basis, all islands (20 of 20) were significantly nested (Table 2). On an individual species basis, 35 of 48 species $(73 \%)$ were significantly nested. Although it was possible to obtain a significant result for all species, power was low for some. If species present on $\geq 18$ or $<2$ islands are excluded, 28 of 32 species ( $88 \%$ ) were significantly nested (Table 2). 
TABLE 1

Occurrences of Ant Species on 14 Hawaiian Islands

\begin{tabular}{|c|c|c|c|c|c|c|c|c|c|c|c|c|c|c|c|c|}
\hline Species/Island ${ }^{a}$ & $\mathrm{Oa}$ & $\mathrm{Ha}$ & $\mathrm{Ka}$ & $\mathrm{Ma}$ & Mo & Ln & Md & Ly & $\mathrm{FF}$ & $\mathrm{Ku}$ & No & $\mathrm{Ne}$ & $\mathrm{Nu}$ & $\mathrm{PH}$ & Incidence & $P$ \\
\hline Cardiocondyla nuda (Mayr) & $\mathrm{X}$ & $\mathrm{X}$ & $\mathrm{X}$ & $\mathrm{X}$ & $\mathrm{X}$ & $\mathrm{X}$ & $\mathrm{X}$ & $\mathrm{X}$ & $\mathrm{X}$ & $\mathrm{X}$ & & $\mathrm{X}$ & & $\mathrm{X}$ & 12 & .071 \\
\hline $\begin{array}{l}\text { Tetramorium bicarinatum } \\
\text { (Nylander) }\end{array}$ & $\mathrm{X}$ & $\mathrm{X}$ & $X$ & $\mathrm{X}$ & $\mathrm{X}$ & & $\mathrm{X}$ & $\mathrm{X}$ & $\mathrm{X}$ & $\mathrm{X}$ & $\mathrm{X}$ & $\mathrm{X}$ & & $\mathrm{X}$ & 12 & .261 \\
\hline Hypoponera punctatissima (Roger) & $\mathrm{X}$ & $\mathrm{X}$ & $\mathrm{X}$ & $\mathrm{X}$ & $\mathrm{X}$ & $\mathrm{X}$ & & $\mathrm{X}$ & $\mathrm{X}$ & $X$ & & $\mathrm{X}$ & & & 10 & .023 \\
\hline Monomorium floricola (Jerdon) & $\mathrm{X}$ & $\mathrm{X}$ & $\mathrm{X}$ & $\mathrm{X}$ & $\mathrm{X}$ & $\mathrm{X}$ & $\mathrm{X}$ & $\mathrm{X}$ & $\mathrm{X}$ & & $\mathrm{X}$ & & & & 10 & .003 \\
\hline Tapinoma melanocephalum (Fabr.) & $\mathrm{X}$ & $\mathrm{X}$ & $\mathrm{X}$ & $\mathrm{X}$ & $\mathrm{X}$ & $\mathrm{X}$ & $\mathrm{X}$ & $\mathrm{X}$ & & $\mathrm{X}$ & $\mathrm{X}$ & & & & 10 & .006 \\
\hline Pheidole megacephala (Fabr.) & $\mathrm{X}$ & $\mathrm{X}$ & $\mathrm{X}$ & $\mathrm{X}$ & $\mathrm{X}$ & $\mathrm{X}$ & $\mathrm{X}$ & $\mathrm{X}$ & & & & & $\mathrm{X}$ & $\mathrm{X}$ & 10 & .038 \\
\hline Camponotus variegatus (F. Smith) & $\mathrm{X}$ & $\mathrm{X}$ & $\mathrm{X}$ & $\mathrm{X}$ & $\mathrm{X}$ & $\mathrm{X}$ & $\mathrm{X}$ & $\mathrm{X}$ & & & & & $\mathrm{X}$ & & 9 & .006 \\
\hline Paratrechina longicornis (Latreille) & $\mathrm{X}$ & $\mathrm{X}$ & $\mathrm{X}$ & $\mathrm{X}$ & $\mathrm{X}$ & $\mathrm{X}$ & $\mathrm{X}$ & & $\mathrm{X}$ & & $\mathrm{X}$ & & & & 9 & .004 \\
\hline Monomorium liliuokalanii Forel & $\mathrm{X}$ & $\mathrm{X}$ & $\mathrm{X}$ & $\mathrm{X}$ & $\mathrm{X}$ & & $\mathrm{X}$ & $\mathrm{X}$ & & & & $\mathrm{X}$ & & & 8 & .012 \\
\hline Plagiolepis alluaudi Emery & $\mathrm{X}$ & $\mathrm{X}$ & $\mathrm{X}$ & $\mathrm{X}$ & $\mathrm{X}$ & $\mathrm{X}$ & $\mathrm{X}$ & $\mathrm{X}$ & & & & & & & 8 & .001 \\
\hline Cardiocondyla venustula Wheeler & $\mathrm{X}$ & $\mathrm{X}$ & $\mathrm{X}$ & $\mathrm{X}$ & $\mathrm{X}$ & $\mathrm{X}$ & & & & & $\mathrm{X}$ & & & & 7 & .004 \\
\hline Hypoponera opaciceps (Mayr) & $\mathrm{X}$ & $\mathrm{X}$ & $\mathrm{X}$ & $\mathrm{X}$ & $\mathrm{X}$ & $\mathrm{X}$ & & & & & & & $\mathrm{X}$ & & 7 & .008 \\
\hline Leptogenys falcigera Roger & $\mathrm{X}$ & $\mathrm{X}$ & $\mathrm{X}$ & $\mathrm{X}$ & $\mathrm{X}$ & $\mathrm{X}$ & & & & & & & $\mathrm{X}$ & & 7 & .008 \\
\hline Paratrechina bourbonica (Forel) & $\mathrm{X}$ & $\mathrm{X}$ & $\mathrm{X}$ & $\mathrm{X}$ & $\mathrm{X}$ & $\mathrm{X}$ & $\mathrm{X}$ & & & & & & & & 7 & .001 \\
\hline Solenopsis geminata (Fabr.) & $\mathrm{X}$ & $\mathrm{X}$ & $\mathrm{X}$ & $\mathrm{X}$ & $\mathrm{X}$ & $\mathrm{X}$ & $\mathrm{X}$ & & & & & & & & 7 & .001 \\
\hline Cardiocondyla emeryi Forel & $\mathrm{X}$ & $\mathrm{X}$ & $\mathrm{X}$ & $\mathrm{X}$ & $\mathrm{X}$ & $\mathrm{X}$ & & & & & & & & & 6 & .001 \\
\hline Cardiocondyla wroughtonii (Forel) & $\mathrm{X}$ & $\mathrm{X}$ & $\mathrm{X}$ & $\mathrm{X}$ & $\mathrm{X}$ & $\mathrm{X}$ & & & & & & & & & 6 & .001 \\
\hline Linepithema bumile (Mayr) & $\mathrm{X}$ & $\mathrm{X}$ & $\mathrm{X}$ & $\mathrm{X}$ & $\mathrm{X}$ & $\mathrm{X}$ & & & & & & & & & 6 & .001 \\
\hline Paratrechina vaga (Forel) & $\mathrm{X}$ & $\mathrm{X}$ & $\mathrm{X}$ & $\mathrm{X}$ & $\mathrm{X}$ & $\mathrm{X}$ & & & & & & & & & 6 & .001 \\
\hline Solenopsis papuana Emery & $\mathrm{X}$ & $\mathrm{X}$ & $\mathrm{X}$ & $\mathrm{X}$ & $\mathrm{X}$ & $\mathrm{X}$ & & & & & & & & & 6 & .001 \\
\hline Tetramorium tonganum Mayr & $\mathrm{X}$ & $\mathrm{X}$ & $\mathrm{X}$ & $\mathrm{X}$ & & & & & $\mathrm{X}$ & $\mathrm{X}$ & & & & & 6 & .023 \\
\hline Cerapachys biroi Forel & $\mathrm{X}$ & $\mathrm{X}$ & $\mathrm{X}$ & $\mathrm{X}$ & $\mathrm{X}$ & & & & & & & & & & 5 & .002 \\
\hline Monomorium destructor (Jerdon) & $\mathrm{X}$ & $\mathrm{X}$ & $\mathrm{X}$ & & & & & $\mathrm{X}$ & $\mathrm{X}$ & & & & & & 5 & .027 \\
\hline Monomorium sechellense Emery & $\mathrm{X}$ & $\mathrm{X}$ & $\mathrm{X}$ & $\mathrm{X}$ & & $\mathrm{X}$ & & & & & & & & & 5 & .002 \\
\hline Monomorium pharaonis (Linné) & $\mathrm{X}$ & $\mathrm{X}$ & $\mathrm{X}$ & $\mathrm{X}$ & & & & & $\mathrm{X}$ & & & & & & 5 & .007 \\
\hline Strumigenys rogeri Emery & $\mathrm{X}$ & $\mathrm{X}$ & $\mathrm{X}$ & $\mathrm{X}$ & $\mathrm{X}$ & & & & & & & & & & 5 & .002 \\
\hline Technomyrmex albipes (F. Smith) & $\mathrm{X}$ & $\mathrm{X}$ & $\mathrm{X}$ & $\mathrm{X}$ & $\mathrm{X}$ & & & & & & & & & & 5 & .002 \\
\hline Tetramorium simillimum (F. Smith) & $\mathrm{X}$ & $\mathrm{X}$ & $\mathrm{X}$ & $\mathrm{X}$ & & $\mathrm{X}$ & & & & & & & & & 5 & .002 \\
\hline Anoplolepis gracilipes (F. Smith) & $\mathrm{X}$ & $\mathrm{X}$ & $\mathrm{X}$ & $\mathrm{X}$ & & & & & & & & & & & 4 & .002 \\
\hline Ochetellus glaber (Mayr) & $\mathrm{X}$ & $\mathrm{X}$ & $\mathrm{X}$ & $X$ & & & & & & & & & & & 4 & .002 \\
\hline Pheidole fervens $\mathrm{F}$. Smith & $\mathrm{X}$ & $\mathrm{X}$ & $\mathrm{X}$ & $\mathrm{X}$ & & & & & & & & & & & 4 & .002 \\
\hline Ponera swezeyi (Wheeler) & $\mathrm{X}$ & $\mathrm{X}$ & $\mathrm{X}$ & $\mathrm{X}$ & & & & & & & & & & & 4 & .002 \\
\hline Strumigenys emmae (Emery) & $\mathrm{X}$ & $\mathrm{X}$ & $\mathrm{X}$ & $\mathrm{X}$ & & & & & & & & & & & 4 & .002 \\
\hline Strumigenys godeffroyi Mayr & $\mathrm{X}$ & $\mathrm{X}$ & $\mathrm{X}$ & $\mathrm{X}$ & & & & & & & & & & & 4 & .002 \\
\hline $\begin{array}{l}\text { Hypoponera zwaluwenburgi } \\
\text { (Wheeler) }\end{array}$ & $\mathrm{X}$ & $\mathrm{X}$ & & $X$ & & & & & & & & & & & 3 & .008 \\
\hline Solenopsis sp. & $\mathrm{X}$ & $\mathrm{X}$ & & & & & & & & & & & & & 2 & .014 \\
\hline Pyramica membranifera (Emery) & $\mathrm{X}$ & $\mathrm{X}$ & & & & & & & & & & & & & 2 & .014 \\
\hline Wasmannia auropunctata (Roger) & & $\mathrm{X}$ & $\mathrm{X}$ & & & & & & & & & & & & 2 & .034 \\
\hline $\begin{array}{l}\text { Amblyopone zwaluwenburgi } \\
\text { (Williams) }\end{array}$ & $\mathrm{X}$ & & & & & & & & & & & & & & 1 & $.053^{b}$ \\
\hline Brachymyrmex obscurior Forel & $\mathrm{X}$ & & & & & & & & & & & & & & 1 & $.053^{b}$ \\
\hline Lepisiota sp. & $\mathrm{X}$ & & & & & & & & & & & & & & 1 & $.053^{b}$ \\
\hline Pheidole moerens Wheeler & & $\mathrm{X}$ & & & & & & & & & & & & & 1 & $.086^{b}$ \\
\hline Pseudomyrmex gracilis (Fabr.) & $\mathrm{X}$ & & & & & & & & & & & & & & 1 & $.053^{b}$ \\
\hline Strumigenys lewisi Cameron & $\mathrm{X}$ & & & & & & & & & & & & & & 1 & $.053^{b}$ \\
\hline Tetramorium insolens (F. Smith) & & $\mathrm{X}$ & & & & & & & & & & & & & 1 & $.086^{b}$ \\
\hline Total species richness & 42 & 40 & 35 & 34 & 23 & 20 & 11 & 10 & 8 & 5 & 5 & 4 & 4 & 3 & & \\
\hline Statistical significance $^{c}$ & ** & $* * *$ & $* * *$ & $* * *$ & $* * *$ & $* * *$ & $* * *$ & $* * *$ & ** & $* * *$ & $* * *$ & ** & * & ** & & \\
\hline
\end{tabular}

${ }^{a}$ Island abbreviations: Oa, O'ahu; Ha, Hawai'i (the Big Island); Ma, Maui; Ka, Kaua'i; Mo, Moloka'i; Ln, Lāna'i; Ly, Laysan; Md, Midway; FF, French Frigate Shoals; Ku, Kure (Ocean); No, Nīhoa; Nu, Ni'ihau; Ne, Necker; PH, Pearl and Hermes Reef. ${ }^{b}$ Not possible to achieve statistical significance at $\alpha=.05$.

${ }^{* * *}, P \leq .001 ;{ }^{* *}, P \leq .01 ;{ }^{*}, P<.05$. 
TABLE 2

Occurrences of Ant Species on 20 Islands in French Polynesia and the Cook Islands

\begin{tabular}{|c|c|c|c|c|c|c|c|c|c|c|c|c|c|c|c|c|c|c|c|c|c|c|}
\hline Species/Island $^{a}$ & $\mathrm{Ta}$ & Mo & $\mathrm{Hu}$ & Bo & $\mathrm{Rt}$ & $\mathrm{Fh}$ & Ho & $\mathrm{Mr}$ & - Ak & Th & $\mathrm{Fg}$ & $\mathrm{Fr}$ & At & Ro & $\mathrm{Mp}$ & Mt & $\mathrm{Ah}$ & $\mathrm{Fr}$ & Ir $B$ & $\mathrm{Bm}$ & $\begin{array}{l}\text { Inci- } \\
\text { dence }\end{array}$ & $P$ \\
\hline $\begin{array}{l}\text { Paratrechina } \\
\quad \text { bourbonica (Forel) }\end{array}$ & $X$ & $\mathrm{X}$ & $X$ & $\mathrm{X}$ & $\mathrm{X}$ & $\mathrm{X}$ & $\mathrm{X}$ & $X$ & $X$ & $\mathrm{X}$ & $\mathrm{X}$ & $\mathrm{X}$ & $\mathrm{X}$ & $\mathrm{X}$ & $X$ & $\mathrm{X}$ & $X$ & & $X$ & $\mathrm{X}$ & 19 & .082 \\
\hline $\begin{array}{l}\text { Paratrechina vaga } \\
\text { (Forel) }\end{array}$ & $X$ & $\mathrm{X}$ & $X$ & $\mathrm{X}$ & $\mathrm{X}$ & $\mathrm{X}$ & $X$ & $X$ & $X$ & $X$ & & $X$ & $X$ & $X$ & $X$ & & $\mathrm{X}$ & $X$ & $\mathrm{X}$ & $X$ & 18 & .205 \\
\hline $\begin{array}{l}\text { Tapinoma } \\
\text { melanocephalum } \\
\text { (Fabr.) }\end{array}$ & $X$ & $X$ & $X$ & $\mathrm{X}$ & $\mathrm{X}$ & $X$ & $X$ & & $X$ & $X$ & $\mathrm{X}$ & $X$ & $\mathrm{X}$ & $\mathrm{X}$ & $X$ & $\mathrm{X}$ & $\mathrm{X}$ & $\mathrm{X}$ & $X$ & & 18 & .188 \\
\hline $\begin{array}{l}\text { Tetramorium } \\
\text { simillimum (F. } \\
\text { Smith) }\end{array}$ & $X$ & $X$ & $X$ & $\mathrm{X}$ & $\mathrm{X}$ & $\mathrm{X}$ & $X$ & $\mathrm{X}$ & $X$ & $\mathrm{X}$ & $\mathrm{X}$ & $X$ & $\mathrm{X}$ & $\mathrm{X}$ & $\mathrm{X}$ & $\mathrm{X}$ & & $X$ & & & 17 & .009 \\
\hline $\begin{array}{l}\text { Solenopsis geminata } \\
\text { (Fabr.) }\end{array}$ & $X$ & X & X & $\mathrm{X}$ & $\mathrm{X}$ & & $\mathrm{X}$ & $\mathrm{X}$ & & $\mathrm{X}$ & $\mathrm{X}$ & $\mathrm{X}$ & $\mathrm{X}$ & & & $X$ & $\mathrm{X}$ & $X$ & $X$ & $\mathrm{X}$ & 16 & .335 \\
\hline $\begin{array}{l}\text { Monomorium floricola } \\
\text { (Jerdon) }\end{array}$ & $X$ & X & X & $X$ & $\mathrm{X}$ & $X$ & $X$ & $\mathrm{X}$ & $\mathrm{X}$ & $X$ & $\mathrm{X}$ & $X$ & $X$ & & $X$ & & $X$ & & X & & 16 & .005 \\
\hline $\begin{array}{l}\text { Anoplolepis gracilipes } \\
\text { (F. Smith) }\end{array}$ & $X$ & $\mathrm{X}$ & $X$ & $\mathrm{X}$ & $\mathrm{X}$ & $\mathrm{X}$ & $X$ & $X$ & $X$ & $\mathrm{X}$ & & $\mathrm{X}$ & $\mathrm{X}$ & & & & & $X$ & & & 13 & .001 \\
\hline $\begin{array}{l}\text { Tetramorium } \\
\text { bicarinatum } \\
\text { (Nylander) }\end{array}$ & $X$ & $X$ & $X$ & $X$ & & $X$ & $X$ & $X$ & $X$ & & $X$ & & $X$ & $X$ & $X$ & & & & & & 12 & .009 \\
\hline $\begin{array}{l}\text { Monomorium } \\
\text { liliuokalanii Forel }\end{array}$ & $X$ & $X$ & $X$ & $X$ & & & $X$ & & $X$ & $X$ & & & & & & $X$ & $X$ & & $X$ & $X$ & 11 & .200 \\
\hline $\begin{array}{l}\text { Paratrechina longicornis } \\
\text { (Latreille) }\end{array}$ & $\mathrm{X}$ & $X$ & $X$ & $X$ & $\mathrm{X}$ & $X$ & $X$ & & & & & & & $X$ & & $X$ & $X$ & $X$ & & & 11 & .043 \\
\hline $\begin{array}{l}\text { Pheidole fervens } \mathrm{F} \text {. } \\
\text { Smith }\end{array}$ & $X$ & $X$ & $X$ & X & $\mathrm{X}$ & $X$ & $X$ & $X$ & $X$ & & & & $X$ & $X$ & & & & & & & 11 & .001 \\
\hline $\begin{array}{l}\text { Pheidole umbonata } \\
\text { Mayr }\end{array}$ & $X$ & $X$ & $X$ & X & $\mathrm{X}$ & $X$ & $X$ & & & $X$ & & $X$ & & & $X$ & & & & & & 10 & .001 \\
\hline $\begin{array}{l}\text { Technomyrmex albipes } \\
\text { (F. Smith) }\end{array}$ & $X$ & $X$ & $X$ & $\mathrm{X}$ & $X$ & X & $X$ & $X$ & $X$ & $X$ & & & & & & & & & & & 10 & $<.001$ \\
\hline $\begin{array}{l}\text { Cardiocondyla } \\
\text { wrougbtonii (Forel) }\end{array}$ & $X$ & $\mathrm{X}$ & $\mathrm{X}$ & $X$ & & $\mathrm{X}$ & $\mathrm{X}$ & $\mathrm{X}$ & $\mathrm{X}$ & & & & & & & $\mathrm{X}$ & & & & & 9 & .002 \\
\hline $\begin{array}{l}\text { Cardiocondyla nuda } \\
\text { (Mayr) }\end{array}$ & $\mathrm{X}$ & $X$ & $X$ & $\mathrm{X}$ & & & & & & $X$ & X & $X$ & & & & & & & & X & 8 & .048 \\
\hline $\begin{array}{l}\text { Tapinoma minutum } \\
\text { Mayr }\end{array}$ & & $X$ & $X$ & $X$ & & $X$ & & & & $X$ & & $\mathrm{X}$ & & & & & $X$ & & $X$ & & 8 & .130 \\
\hline $\begin{array}{l}\text { Plagiolepis alluaudi } \\
\text { Emery }\end{array}$ & $X$ & $X$ & $X$ & $X$ & & & $X$ & $X$ & & & & & & & & $X$ & & & & & 7 & .004 \\
\hline Pheidole oceanica Mayr & $\mathrm{X}$ & $X$ & $X$ & & $X$ & & $X$ & & $X$ & & & & & & $X$ & & & & & & 7 & .008 \\
\hline $\begin{array}{l}\text { Tetramorium pacificum } \\
\text { Mayr }\end{array}$ & $\mathrm{X}$ & $\mathrm{X}$ & $\mathrm{X}$ & $\mathrm{X}$ & $\mathrm{X}$ & $\mathrm{X}$ & & $\mathrm{X}$ & & & & & & & & & & & & & 7 & $<.001$ \\
\hline $\begin{array}{l}\text { Tetramorium } \\
\text { tonganum Mayr }\end{array}$ & $X$ & X & $\mathrm{X}$ & X & $X$ & $X$ & $\mathrm{X}$ & & & & & & & & & & & & & & 7 & $<.001$ \\
\hline $\begin{array}{l}\text { Strumigenys godeffroyi } \\
\text { Mayr }\end{array}$ & $\mathrm{X}$ & X & $X$ & X & X & $\mathrm{X}$ & & $X$ & & & & & & & & & & & & & 7 & $<.001$ \\
\hline $\begin{array}{l}\text { Pheidole megacephala } \\
\text { (Fabr.) }\end{array}$ & $X$ & & & & $\mathrm{X}$ & $X$ & $X$ & $X$ & $X$ & & & & $X$ & & & & & & & & 7 & .028 \\
\hline $\begin{array}{l}\text { Cardiocondyla emeryi } \\
\text { Forel }\end{array}$ & $X$ & $X$ & $X$ & $X$ & & & & & & $X$ & & $X$ & & & & & & & & & 6 & .004 \\
\hline $\begin{array}{l}\text { Tetramorium } \\
\text { caldarium (Roger) }\end{array}$ & & $X$ & $X$ & $X$ & & $X$ & $X$ & $X$ & & & & & & & & & & & & & 6 & .003 \\
\hline $\begin{array}{l}\text { Strumigenys rogeri } \\
\text { Emery }\end{array}$ & $X$ & $X$ & $X$ & $X$ & & & & $X$ & & & & & & & & & & & & & 5 & .001 \\
\hline $\begin{array}{l}\text { Hypoponera opaciceps } \\
\text { (Mayr) }\end{array}$ & $X$ & $X$ & $X$ & $\mathrm{X}$ & & & & & & & $X$ & & & & & & & & & & 5 & .004 \\
\hline
\end{tabular}


TABLE 2 (continued)

\begin{tabular}{|c|c|c|c|c|c|c|c|c|c|c|c|c|c|c|c|c|c|c|c|c|}
\hline Species/Island ${ }^{a}$ & Ta & Mo & $\mathrm{Hu}$ & Bo & $\mathrm{Rt}$ & $\mathrm{Fh}$ & Ho & $\mathrm{Mr}$ & $\mathrm{Ak}$ & Th & $\mathrm{Fg}$ & Fr At & Ro & $M p$ & Mt & $\mathrm{Ah}$ & Fr Ir & $\mathrm{Bm}$ & $\begin{array}{l}\text { Inci- } \\
\text { dence }\end{array}$ & $P$ \\
\hline $\begin{array}{l}\text { Solenopsis papuana } \\
\text { Emery }\end{array}$ & $\mathrm{X}$ & $\mathrm{X}$ & $X$ & $\mathrm{X}$ & $X$ & & & & & & & & & & & & & & 5 & $<.001$ \\
\hline $\begin{array}{l}\text { Monomorium talpa } \\
\text { Emery }\end{array}$ & & $\mathrm{X}$ & $X$ & $X$ & & & & & & & & $X$ & & & & & & & 4 & .018 \\
\hline $\begin{array}{l}\text { Rogeria sublevinodis } \\
\text { Emery }\end{array}$ & $\mathrm{X}$ & $\mathrm{X}$ & $X$ & $\mathrm{X}$ & & & & & & & & & & & & & & & 4 & .001 \\
\hline $\begin{array}{l}\text { Pheidole sexspinosa } \\
\text { Mayr }\end{array}$ & $\mathrm{X}$ & $\mathrm{X}$ & $\mathrm{X}$ & $X$ & & & & & & & & & & & & & & & 4 & .001 \\
\hline $\begin{array}{l}\text { Anochetus graeffei } \\
\text { Mayr }\end{array}$ & $X$ & $\mathrm{X}$ & $\mathrm{X}$ & $X$ & & & & & & & & & & & & & & & 4 & .001 \\
\hline $\begin{array}{l}\text { Strumigenys emmae } \\
\text { (Emery) }\end{array}$ & & $\mathrm{X}$ & & & & & & & & $\mathrm{X}$ & $\mathrm{X}$ & & & & & & & & 3 & .197 \\
\hline $\begin{array}{l}\text { Hypoponera } \\
\text { punctatissima } \\
\text { (Roger) }\end{array}$ & $X$ & $\mathrm{X}$ & & & & & & & & & $\mathrm{X}$ & & & & & & & & 3 & .040 \\
\hline $\begin{array}{l}\text { Pyramica } \\
\text { membranifera } \\
\text { (Emery) }\end{array}$ & $\mathrm{X}$ & $\mathrm{X}$ & $X$ & & & & & & & & & & & & & & & & 3 & .004 \\
\hline $\begin{array}{l}\text { Pachycondyla } \mathrm{cf} . \\
\text { testacea (Bernard) }\end{array}$ & & $\mathrm{X}$ & $\mathrm{X}$ & $\mathrm{X}$ & & & & & & & & & & & & & & & 3 & .009 \\
\hline $\begin{array}{l}\text { Monomorium destructor } \\
\text { (Jerdon) }\end{array}$ & X & & & & $X$ & & & & & & & & & & & & $X$ & & 3 & .262 \\
\hline $\begin{array}{l}\text { Strumigenys mumfordi } \\
\text { (Wheeler) }\end{array}$ & $\mathrm{X}$ & & & & & & & & & & & & & & & & & & 1 & .049 \\
\hline $\begin{array}{l}\text { Oligomyrmex corniger } \\
\text { Forel }\end{array}$ & $\mathrm{X}$ & & & & & & & & & & & & & & & & & & 1 & .049 \\
\hline Ponera bablet $i$ Perrault & & & & & & & & & & & $X$ & & & & & & & & 1 & .397 \\
\hline $\begin{array}{l}\text { Monomorium pharaonis } \\
\text { (Linné) }\end{array}$ & & & & & & $\mathrm{X}$ & & & & & & & & & & & & & 1 & .216 \\
\hline $\begin{array}{l}\text { Monomorium } \\
\text { sechellense Emery }\end{array}$ & & & & & & & & & $\mathrm{X}$ & & & & & & & & & & 1 & .431 \\
\hline Brachymyrmex sp. & $\mathrm{X}$ & & & & & & & & & & & & & & & & & & 1 & .049 \\
\hline $\begin{array}{l}\text { Tetramorium } \\
\quad \text { lanuginosum Mayr }\end{array}$ & & & & & & & & & & & & & $\mathrm{X}$ & & & & & & 1 & .192 \\
\hline $\begin{array}{l}\text { Platythyrea parallela } \\
\text { (F. Smith) }\end{array}$ & $X$ & & & & & & & & & & & & & & & & & & 1 & .049 \\
\hline $\begin{array}{l}\text { Hypoponera confinis } \\
\text { (Roger) }\end{array}$ & $\mathrm{X}$ & & & & & & & & & & & & & & & & & & 1 & .049 \\
\hline $\begin{array}{l}\text { Ponera swezeyi } \\
\text { (Wheeler) }\end{array}$ & $\mathrm{X}$ & & & & & & & & & & & & & & & & & & 1 & .049 \\
\hline $\begin{array}{l}\text { Odontomachus } \\
\text { simillimus F. Smith }\end{array}$ & $X$ & & & & & & & & & & & & & & & & & & 1 & .049 \\
\hline $\begin{array}{l}\text { Rogeria stigmatica } \\
\text { Emery }\end{array}$ & & & & & $\mathrm{X}$ & & & & & & & & & & & & & & 1 & .216 \\
\hline Total species richness & 38 & 34 & 32 & 30 & 19 & 19 & 19 & 16 & 14 & 14 & 11 & 1210 & 8 & 8 & 8 & 8 & 77 & 5 & & \\
\hline $\begin{array}{l}\text { Statistical } \\
\text { significance }^{b}\end{array}$ & * & ** & ** & ** & $* *$ & $* *$ & ** & $* *$ & $* *$ & $* *$ & * & $* * \quad * *$ & $* *$ & $* *$ & $* *$ & $* *$ & $* * * *$ & $* * *$ & & \\
\hline
\end{tabular}

${ }^{a}$ Island abbreviations: Ta, Tahiti; Mo, Moorea; Hu, Huahine; Bo, Bora Bora; Rt, Rarotonga; Fh, Fatu Hiva; Ho, Hiva Oa; Mr, Mangareva; Ak, Aitutaki; Th, Tiahura; Fg, Fangataufa; Fr, Fareone; At, Aitu; Ro, Raroia; Mp, Maupihaa; Mt, Mataiva; Ah, Ahi; Fr, Fakarava; Ir, Irioa; Bm, Bora Bora motu (unnamed islet on the Bora Bora barrier reef).

$b * *, P \leq .001 ;{ }^{*}, P \leq .01$. 
TABLE 3

Occurrences of Ant Species on Nine of the Most Thoroughly Collected Islands in French Polynesia

\begin{tabular}{|c|c|c|c|c|c|c|c|c|c|c|c|}
\hline Species/Island $^{a}$ & Ta & Mo & $\mathrm{Hu}$ & Bo & Th & Fr & $\mathrm{Ah}$ & Ir & $\mathrm{Bm}$ & Incidence & $P$ \\
\hline Paratrechina bourbonica (Forel) & $\mathrm{X}$ & $\mathrm{X}$ & $\mathrm{X}$ & $\mathrm{X}$ & $\mathrm{X}$ & $\mathrm{X}$ & $\mathrm{X}$ & $\mathrm{X}$ & $\mathrm{X}$ & 9 & \\
\hline Paratrechina vaga (Forel) & $X$ & $X$ & $\mathrm{X}$ & $\mathrm{X}$ & $\mathrm{X}$ & $\mathrm{X}$ & $\mathrm{X}$ & $\mathrm{X}$ & $\mathrm{X}$ & 9 & \\
\hline Solenopsis geminata (Fabr.) & $X$ & $\mathrm{X}$ & $X$ & $\mathrm{X}$ & $X$ & $\mathrm{X}$ & $\mathrm{X}$ & $\mathrm{X}$ & $\mathrm{X}$ & 9 & \\
\hline Monomorium liliuokalanii Forel & $\mathrm{X}$ & $\mathrm{X}$ & $\mathrm{X}$ & $\mathrm{X}$ & $\mathrm{X}$ & & $\mathrm{X}$ & $\mathrm{X}$ & $X$ & 8 & $.350^{b}$ \\
\hline Tapinoma melanocephalum (Fabr.) & $\mathrm{X}$ & $\mathrm{X}$ & $\mathrm{X}$ & $\mathrm{X}$ & $\mathrm{X}$ & $\mathrm{X}$ & $\mathrm{X}$ & $\mathrm{X}$ & & 8 & 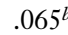 \\
\hline Monomorium floricola (Jerdon) & $\mathrm{X}$ & $\mathrm{X}$ & $\mathrm{X}$ & $\mathrm{X}$ & $\mathrm{X}$ & $\mathrm{X}$ & $\mathrm{X}$ & $\mathrm{X}$ & & 8 & $.065^{b}$ \\
\hline Cardiocondyla nuda (Mayr) & $\mathrm{X}$ & $\mathrm{X}$ & $\mathrm{X}$ & $\mathrm{X}$ & $\mathrm{X}$ & $\mathrm{X}$ & & & $X$ & 7 & .072 \\
\hline Tapinoma minutum Mayr & & $\mathrm{X}$ & $\mathrm{X}$ & $\mathrm{X}$ & $X$ & $\mathrm{X}$ & $\mathrm{X}$ & $\mathrm{X}$ & & 7 & .500 \\
\hline Tetramorium simillimum (F. Smith) & $\mathrm{X}$ & $\mathrm{X}$ & $\mathrm{X}$ & $\mathrm{X}$ & $\mathrm{X}$ & $\mathrm{X}$ & & & & 6 & .010 \\
\hline Pheidole umbonata Mayr & $\mathrm{X}$ & $\mathrm{X}$ & $\mathrm{X}$ & $\mathrm{X}$ & $\mathrm{X}$ & $\mathrm{X}$ & & & & 6 & .010 \\
\hline Anoplolepis gracilipes (F. Smith) & $\mathrm{X}$ & $\mathrm{X}$ & $\mathrm{X}$ & $\mathrm{X}$ & $\mathrm{X}$ & $\mathrm{X}$ & & & & 6 & .010 \\
\hline Cardiocondyla emeryi Forel & $\mathrm{X}$ & $\mathrm{X}$ & $\mathrm{X}$ & $X$ & $\mathrm{X}$ & $\mathrm{X}$ & & & & 6 & .010 \\
\hline Paratrechina longicornis (Latreille) & $\mathrm{X}$ & $\mathrm{X}$ & $\mathrm{X}$ & $X$ & & & $\mathrm{X}$ & & & 5 & .025 \\
\hline Technomyrmex albipes (F. Smith) & $\mathrm{X}$ & $\mathrm{X}$ & $\mathrm{X}$ & $\mathrm{X}$ & $\mathrm{X}$ & & & & & 5 & .007 \\
\hline Monomorium talpa Emery & & $\mathrm{X}$ & $\mathrm{X}$ & $X$ & & $\mathrm{X}$ & & & & 4 & .111 \\
\hline Pheidole fervens $\mathrm{F}$. Smith & $\mathrm{X}$ & $\mathrm{X}$ & $\mathrm{X}$ & $\mathrm{X}$ & & & & & & 4 & .007 \\
\hline Tetramorium bicarinatum (Nylander) & $\mathrm{X}$ & $\mathrm{X}$ & $\mathrm{X}$ & $\mathrm{X}$ & & & & & & 4 & .007 \\
\hline Cardiocondyla wroughtonii (Forel) & $\mathrm{X}$ & $\mathrm{X}$ & $\mathrm{X}$ & $\mathrm{X}$ & & & & & & 4 & .007 \\
\hline Plagiolepis alluaudi Emery & $\mathrm{X}$ & $\mathrm{X}$ & $\mathrm{X}$ & $\mathrm{X}$ & & & & & & 4 & .007 \\
\hline Tetramorium pacificum Mayr & $\mathrm{X}$ & $\mathrm{X}$ & $\mathrm{X}$ & $\mathrm{X}$ & & & & & & 4 & .007 \\
\hline Tetramorium tonganum Mayr & $\mathrm{X}$ & $\mathrm{X}$ & $\mathrm{X}$ & $X$ & & & & & & 4 & .007 \\
\hline Strumigenys godeffroyi Mayr & $\mathrm{X}$ & $\mathrm{X}$ & $\mathrm{X}$ & $\mathrm{X}$ & & & & & & 4 & .007 \\
\hline Strumigenys rogeri Emery & $\mathrm{X}$ & $\mathrm{X}$ & $\mathrm{X}$ & $\mathrm{X}$ & & & & & & 4 & .007 \\
\hline Hypoponera opaciceps (Mayr) & $\mathrm{X}$ & $\mathrm{X}$ & $\mathrm{X}$ & $\mathrm{X}$ & & & & & & 4 & .007 \\
\hline Solenopsis papuana Emery & $\mathrm{X}$ & $\mathrm{X}$ & $\mathrm{X}$ & $\mathrm{X}$ & & & & & & 4 & .007 \\
\hline Rogeria sublevinodis Emery & $\mathrm{X}$ & $\mathrm{X}$ & $\mathrm{X}$ & $\mathrm{X}$ & & & & & & 4 & .007 \\
\hline Pheidole sexspinosa Mayr & $\mathrm{X}$ & $\mathrm{X}$ & $\mathrm{X}$ & $\mathrm{X}$ & & & & & & 4 & .007 \\
\hline Anochetus graeffei Mayr & $X$ & $\mathrm{X}$ & $\mathrm{X}$ & $X$ & & & & & & 4 & .007 \\
\hline Tetramorium caldarium (Roger) & & $\mathrm{X}$ & $\mathrm{X}$ & $\mathrm{X}$ & & & & & & 3 & .061 \\
\hline Pachycondyla cf. testacea (Bernard) & & $\mathrm{X}$ & $\mathrm{X}$ & $\mathrm{X}$ & & & & & & 3 & .061 \\
\hline Pheidole oceanica Mayr & $\mathrm{X}$ & $\mathrm{X}$ & $\mathrm{X}$ & & & & & & & 3 & .010 \\
\hline Pyramica membranifera (Emery) & $X$ & $X$ & $X$ & & & & & & & 3 & .010 \\
\hline Strumigenys emmae (Emery) & & $\mathrm{X}$ & & & $\mathrm{X}$ & & & & & 2 & .190 \\
\hline Hypoponera punctatissima (Roger) & $X$ & $\mathrm{X}$ & & & & & & & & 2 & .020 \\
\hline Strumigenys mumfordi (Wheeler) & $\mathrm{X}$ & & & & & & & & & 1 & 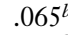 \\
\hline Pheidole megacephala (Fabr.) & $\mathrm{X}$ & & & & & & & & & 1 & 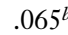 \\
\hline Oligomyrmex corniger Forel & $\mathrm{X}$ & & & & & & & & & 1 & 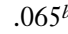 \\
\hline Monomorium destructor (Jerdon) & $\mathrm{X}$ & & & & & & & & & 1 & 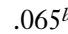 \\
\hline Brachymyrmex sp. & $\mathrm{X}$ & & & & & & & & & 1 & 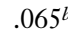 \\
\hline Platythyrea parallela (F. Smith) & $\mathrm{X}$ & & & & & & & & & 1 & 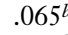 \\
\hline Hypoponera confinis (Roger) & $\mathrm{X}$ & & & & & & & & & 1 & 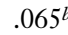 \\
\hline Ponera swezeyi (Wheeler) & $\mathrm{X}$ & & & & & & & & & 1 & 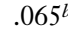 \\
\hline Odontomachus simillimus $\mathrm{F}$. Smith & $\mathrm{X}$ & & & & & & & & & 1 & 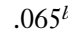 \\
\hline Total species richness & 38 & 34 & 32 & 30 & 14 & 12 & 8 & 7 & 5 & & \\
\hline Statistical significance ${ }^{c}$ & ns & ** & ** & ** & ** & ** & ** & ** & ** & & \\
\hline
\end{tabular}

a Island abbreviations: Ta, Tahiti; Mo, Moorea; Hu, Huahine; Bo, Bora Bora; Th, Tiahura; Fr, Faerone; Ah, Ahi; Ir, Irioa; Bm, Bora Bora motu (unnamed islet on the barrier reef of Bora Bora).

${ }^{b}$ Not possible to achieve statistical significance at $\alpha=.05$.

${ }^{* *}, P \leq .001 ; \mathrm{ns}$, not significant $(P<.05)$.

In the subset of nine islands in the French Polynesia/Cook Island group that I consider to be thoroughly collected, eight of the nine $(89 \%)$ islands (all except Tahiti) were signifi- cantly nested on an individual island basis (Table 3). Because there were only nine islands, a significant result could not be obtained for species that occurred on eight, 
nine, or only one island. Thus, on an individual species basis, 22 of 28 species (79\%) for which a significant result could be obtained were significantly nested. Even though not all species could be statistically tested, examination of Table 3 by eye reveals a strong degree of nestedness.

\section{DISCUSSION}

\section{Nestedness}

A number of indices that generate a single statistic to measure the nestedness of an entire biotic assemblage have been proposed (Cook 1995). The advantages of the method used here are that it allows the assessment of nestedness for individual species as well as the biotic assemblage as a whole, aspects of its statistical properties are well known, and computer simulations are not required to determine significance levels (Simberloff and Martin 1991). Most biotic assemblages as a whole, when tested for nestedness, have been shown to exhibit significant nestedness patterns (Cook and Quinn 1995). It has thus been argued that the most informative question is not whether a biotic assemblage as a whole reveals an overall nestedness pattern, but rather which individual species do not display patterns of nestedness, and why (Simberloff and Martin 1991, Kadmon 1995). Moreover, even though most biotic assemblages as a whole reveal significant nestedness, when species are tested individually a relatively small number may be significantly nested. For example, in a study of plants on islands in a reservoir, Kadmon (1995) found that although the entire assemblage was significantly nested, only a relatively small fraction of the individual species $(<20 \%)$ displayed significant nestedness.

In my analyses, the great majority of individual species $(73-95 \%)$ and individual islands $(89-100 \%)$ were significantly nested. Most that were not had low statistical power to detect a significant degree of nestedness. In the Hawaiian Archipelago, for example, the only species that did not display significant nestedness were those that were present on only one island or those that were present on all but two islands. In both cases statistical power was low. In fact, it was not possible to obtain a "significant" result $(\alpha=.05)$ for species present on only one island.

The most likely explanation for some species in French Polynesia displaying a nonsignificant degree of nestedness (yet with reasonable statistical power to detect it) is that there has not been adequate collecting effort on some of the islands outside the Society group. The reduced Society Island data set, which demonstrates the greatest degree of nesting, was obtained primarily from the records of only two collectors, and species concepts were employed consistently, at least for the eight islands collected by Morrison $(1995,1996 a)$. In this case, nine islands is a relatively small sample size for this type of analysis, and even a slight deviation from a perfectly nested occurrence sequence can result in a nonsignificant result. For example, four of the five species that occurred on between two and seven islands and did not display a significant degree of nestedness ( $T$. minutum, M. talpa, T. caldarium, and P. testacea) were absent from Tahiti (the most speciose island), although the remainder of their occurrence sequences were highly nested. Given the necessity of almost perfectly ordered occurrence sequences to obtain significant results in this reduced set of nine islands, it is somewhat amazing that so many species were found to be significantly nested.

Nestedness may be evaluated by ranking islands according to size, rather than species richness (Simberloff and Martin 1991, Cook 1995). Comparability of the results depends upon the strength of the species-area relationship for the group of islands in question. Species richnesses on these Polynesian islands are strongly positively correlated with area (Hawaiian Islands: $r=0.82, \quad P=.0004$; French Polynesia and the Cook Islands: $r=$ $0.69, P=.0007$; Society Islands: $r=0.97$, $P<.0001$; areas $\log$ transformed before analyses). The correlation for the more thoroughly collected Society Island subset is particularly strong; the relatively weaker correlation for the larger French Polynesia/ Cook Islands group is likely due to less- 
thorough collections on some islands. In general, however, high degrees of nestedness exist regardless of how islands are ranked.

\section{Mechanisms}

In general, insular ant species occurrences are a function of three primary factors: (1) dispersal ability, (2) habitat suitability, and (3) interactions with other species. Dispersal in this system may include both transportation by humans via ships and planes, and natural dispersal by mating flights of reproductives. Individual species likely possess traits that make them more successful at one of the mechanisms of dispersal than the other.

In general, if dispersal ability is of primary importance, one would expect that the better dispersers would be present on more islands. Given the distances separating the more remote Polynesian islands from continental areas and other islands, all ants must first be introduced to Polynesia by humans. Because Tahiti is a hub of commerce for French Polynesia, as $\mathrm{O}^{\prime}$ ahu is for the Hawaiian Islands, more international ship and plane traffic likely explains the higher species richnesses of these two islands relative to the remainder of their respective archipelagoes. Once present on these international "hubs," interisland commerce will allow the spread of ants among the more populated islands. Given the distances separating islands in Polynesia, most ant species probably colonize new islands through human transport mechanisms. Once present in an archipelago, however, species may reach relatively near islands through mating flights of reproductives.

The small islands on the barrier reefs of the Society group are of great interest in addressing the question of natural dispersal. Human transport to these uninhabited islands is unlikely because the only visitors use small boats and carry no cargo. All small islands are relatively close to their respective mainland islands $(\leq 820 \mathrm{~m})$, so distance should not be a barrier to natural dispersal. Almost all ant species should be able to reach these small islands, yet their faunas are highly nested (five least-speciose islands in Table 3).
If suitable habitat determines which species may colonize, larger islands would be expected to have more species because they have more different habitats (e.g., Connor and McCoy 1979). (Tahiti is the largest of the Society Islands, and $\mathrm{O}^{\prime}$ ahu is the third largest of the Hawaiian Islands.) If larger islands have the same types of habitats as smaller ones, plus some additional habitat types, then a nested pattern would be expected. High islands accrue more habitats with increasing size than atolls, which frequently exist as a series of smaller islets. Unfortunately not enough atolls have been thoroughly collected to evaluate this difference.

Six of the seven species commonly found (present on at least four islands) on the five small islands on the barrier reefs of Moorea and Bora Bora displayed a significant preference for disturbed sites on the main island of Moorea (Morrison 1995). Paratrechina vaga, the only exception, was present at the great majority ( $85 \%)$ of sites on Moorea. Thus, ants present on the smallest islands, which could be considered very disturbed due to tides, waves, and winds, are also found in disturbed areas on larger islands. Disturbance on the larger island of Moorea could have been natural (i.e., along the coastline) or humaninduced (i.e., deforestation, agriculture, etc.). Thus almost all islands regardless of size could be expected to possess this subset of species that can survive very disturbed or marginal habitats. Conversely, some species are associated with specialized habitats (e.g., Pachycondyla testacea was found only in moist undisturbed forests in the higher elevations [Morrison 1995]) and would not be present on islands lacking those habitat types. Thus the presence or absence of the appropriate habitat may be an important factor for many species.

Finally, interspecific interactions may affect distribution patterns. As noted previously, in general competitive exclusion probably does not determine species occurrence patterns among these islands (Morrison $1996 a, b)$. An exception to this rule, however, can be found in the introduction of the 
Argentine ant, Linepithema humile, to Easter Island, which apparently led to a lower ant species diversity and potential exclusion of some ant species from the island (Morrison 1997). Linepithema bumile is also present on the larger Hawaiian islands, yet it occurs in the higher elevations (Reimer 1994) and there is no evidence that it has reduced islandwide ant species diversity.

Linepithema bumile is one of several invasive ant species that are known to have strong impacts on the biota of recipient communities (Holway et al. 2002). Another of these species, the little fire ant, Wasmannia auropunctata, has recently been introduced to both the Hawaiian Islands and French Polynesia (Krushelnycky et al. 2005). Although such invasive species have been implicated in the reduction of ant species diversity in invaded communities (Holway et al. 2002), the manner in which they may affect the ant species composition of an entire island, particularly high islands with diverse habitats, remains an open question. Thus overall, with the possible exception of a few particularly aggressive ant species, it appears that dispersal opportunities combined with habitat suitability represent the primary mechanisms underlying the observed patterns.

\section{CONCLUSIONS}

The introduced ant faunas of remote Polynesian islands are not random assortments of the available species pool; they are highly ordered (i.e., nested) assemblages. Contributions to this overall nestedness were made by almost all species that were neither so rare nor so common that statistical power to detect such patterns was low. The degree of nestedness was strong even though some islands have not been thoroughly collected; excluding such islands (in French Polynesia) resulted in even stronger nestedness patterns. These interisland patterns of community assembly, combined with intraisland patterns of ant community structure and function (Morrison 1996b), reveal surprising degrees of order in a fauna that has been introduced recently in evolutionary time, from origins around the world.

\section{Literature Cited}

Connor, E. F., and E. D. McCoy. 1979. The statistics and biology of the species-area relationship. Am. Nat. 113:791-833.

Cook, R. R. 1995. The relationship between nested subsets, habitat subdivision, and species diversity. Oecologia (Berl.) 101: 204-210.

Cook, R. R., and J. F. Quinn. 1995. The influence of colonization in nested species subsets. Oecologia (Berl.) 102: 413-424.

Gruner, D. S. 2003. Two ant species (Hymenoptera: Formicidae) new to the Hawaiian Islands. Bishop Mus. Occas. Pap. 74:3540.

Hecnar, S. J., G. S. Casper, R. W. Russell, D. R. Hecnar, and J. N. Robinson. 2002. Nested species assemblages of amphibians and reptiles on islands in the Laurentian Great Lakes. J. Biogeogr. 29:475-489.

Hecnar, S. J., and R. T. M'Closkey. 1997. Patterns of nestedness and species association in a pond-dwelling amphibian fauna. Oikos 80:371-381.

Hölldobler, B., and E. O. Wilson. 1990. The ants. Belknap Press, Cambridge, Massachusetts.

Holway, D. A., L. Lach, A. V. Saurez, N. D. Tsutsui, and T. J. Case. 2002. The causes and consequences of ant invasions. Annu. Rev. Ecol. Syst. 33:181-233.

Kadmon, R. 1995. Nested species subsets and geographic isolation: A case study. Ecology 76:458-465.

Krushelnycky, P. D., L. L. Loope, and N. J. Reimer. 2005. The ecology, policy, and management of ants in Hawaii. Proc. Hawaii. Entomol. Soc. 37:1-25.

Kumashiro, B. R., R. A. Heu, G. M. Nishida, and J. W. Beardsley. 2001. New state records of immigrant insects in the Hawaiian Islands for the year 1999. Proc. Hawaii. Entomol. Soc. 37:170-184.

Morrison, L. W. 1995. The island biogeography and metapopulation dynamics of ants (Hymenoptera: Formicidae). Ph.D. diss., University of California at Davis, Davis, California.

_ 1996a. The ants (Hymenoptera: For- 
micidae) of Polynesia revisited: Species numbers and the importance of sampling intensity. Ecography 19:73-84.

- 1996 b. Community organization in a recently assembled fauna: The case of Polynesian ants. Oecologia (Berl.) 107: 243-256.

. 1997. Polynesian ant (Hymenoptera: Formicidae) species richness and distribution: A regional survey. Acta Oecol. 18:685-695.

Nishida, G. M., ed. 2002. Hawaiian terrestrial arthropod checklist. 4th ed. Bishop Mus. Tech. Rep. 22. Hawai'i Biological Survey, Bishop Museum, Honolulu, Hawai'i.

Nores, M. 1995. Insular biogeography of birds on mountain-tops in north western Argentina. J. Biogeogr. 22:61-70.

Patterson, B. D. 1984. Mammalian extinction and biogeography in the southern Rocky Mountains. Pages 247-293 in M. H. Nitecki, ed. Extinctions. University of Chicago Press, Chicago.

1987. The principle of nested subsets and its implications for biological conservation. Conserv. Biol. 1:323-334.

Patterson, B. D., and W. Atmar. 1986. Nested subsets and the structure of insular mammalian faunas and archipelagos. Biol. J. Linn. Soc. 28:65-82.

Perrault, G. H. 1988. Les fourmis de Tahiti. Bull. Soc. Zool. Fr. 112:429-445.

- 1993. Peuplement en fourmis de l'atoll de Fangataufa. Bull. Soc. Entomol. Fr. 98:323-338.

Reimer, N. J. 1994. Distribution and impact of alien ants in vulnerable Hawaiian ecosystems. Pages 11-22 in D. F. Williams, ed. Exotic ants: Biology, impact, and con- trol of introduced species. Westview Press, Boulder, Colorado.

Schoener, T. W., and A. Schoener. 1983. Distribution of vertebrates on some very small islands. I. Occurrence sequences of individual species. J. Anim. Ecol. 52:209235.

Seifert, B. 2003. The ant genus Cardiocondyla (Insecta: Hymenoptera: Formicidae)-a taxonomic revision of the $C$. elegans, $C$. bulgarica, C. batesii, C. nuda, C. shuckardi, C. stambuloffii, C. wroughtonii, C. emeryi, and C. minutior species groups. Ann. Naturhist. Mus. Wien Ser. B Bot. Zool. 104:203-338.

Simberloff, D., and B. Levin. 1985. Predictable sequences of species loss with decreasing island area-land birds in two archipelagoes. N. Z. J. Ecol. 8:11-20.

Simberloff, D., and J.-L. Martin. 1991. Nestedness of insular avifaunas: Simple summary statistics masking complex species patterns. Ornis Fenn. 68:178-192.

Sokal, R. R., and F. J. Rohlf. 1995. Biometry. W. H. Freeman and Company, New York. SPSS. 2005. 11.0.4 for Mac OSX. SPSS Inc., Chicago, Illinois.

Wilson, E. O., and R. W. Taylor. 1967a. The ants of Polynesia (Hymenoptera: Formicidae). Pac. Insects Monogr. 14:1-109.

. 1967b. An estimate of the potential evolutionary increase in species density in the Polynesian ant fauna. Evolution 21:110.

Wright, D. H., B. D. Patterson, G. M. Mikkelson, A. Cutler, and W. Atmar. 1998. A comparative analysis of nested subset patterns of species composition. Oecologia (Berl.) 113:1-20. 
